\title{
AYURLOG
}

National Journal of Research in Ayurved Science

http://www.ayurlog.com

Sept- $\left.2020\right|^{\text {Volume } 08^{\text {th }}} \mid$ Issue: $5^{\text {th }}$

ISSN: 2320-7329

\section{A Case Study of Janusandhigatavata (Osteoarthritis of knee joint)}

\section{Anushri Gokul Endait ${ }^{1}$, Mayuri Santosh Nagre², Smita Madhav Kanawade ${ }^{3}$}

1. Assistant Professor Dept. of Panchakarma

2. Assistant Professor Dept. of Kaumarbhritya

3. Assistant Professor Dept. of Shalyatantra

SMBT Ayurveda College \& hospital, Dhamangaon, Nashik, Maharashtra,

\section{*Corresponding Author: anetke939@gmail.com}

\begin{abstract}
-
Osteoarthritis is one of the most common disorders in joint disease. It is characterized by pain, shiftiness, decreased range of motion which is very close to Sandhigatavat. Sandhigatavat is amongst 80 Nanatmaj Vat Vyadhi. Here is a case of 57 years old male patient who was having complaints of pain, tenderness, crepitus, swelling and restricted movement of both knee joints. Based on these symptoms and clinical sign he was diagnosed as a case of Sandhigatvat. Treatment given to this patient was Snehan, Swedan, Janubasti, Yog, Basti and oral medication . Assessment of patient before and after treatment was done on the basis of pain, tenderness and physical activity to exclude the efficacy of the treatment. Based on assessment parameter, moderate improvement was seen in subjective sign \& symptoms after the
\end{abstract}

completion of Schedule of IPD treatment \& 1 month follow up. The pain \& physical activity were improved significantly.

Keywords: Osteoarthritis, Sandhigatavata, Vata, Basti

\section{INTRODUCTION}

In Charak samhita, two types of Vat Vyadhi are mentioned, Nirupstambhit Vat vyadhi that develop due to Dhatukshay \& Upastambhit Vat Vyadhi that develop due to margavarodh due to kapha \& aam. Charakacharya has explained it as 'Sandhigata Anil' which on palpation feels like bag filled with air.

Food, lifestyle \& Environment are three important determinants related to the cause of disease. Nowadays continuous standing work, stress factor; sedentary life leads to Vatprakop which may be due to 
Dhatukshaya (Degeneration) or Margavarodh (obstruction). This Prakupit Vat is extended to the joints where there is kha-vaigunya and marks the beginning of Sandhigatavat. Symptoms of Sandhigatavat are pain, swelling, tenderness and restricted movement of joints. When the decease occurs after $4^{\text {th }}$ decade of life, it is mainly due to Dhatukshaya. According to modern, symptoms of O.A. are loss of articular cartilage, swelling, pain, synovial inflammation, joint shiftiness, loss of mobility and it is a degenerative disease. The comment obstacle for the elderly is to carry out all daily living \& decreased mobility.

Detailed management of this condition in Ayurveda texts contains a combination of external \& internal medication the bahya Chikitsa includes Snehan, Swedan, Janubasti, PatrapindIa etc. Abhyantar Chikitsa include Churna, Kashay, vati, asav arishtas and many more processes.

\section{Materials \& Methods}

A 57-year-old male patient came to Panchakarma OPD at SMBT $\underline{\text { Ayurveda }}$ College \& hospital, Dhamangaon, Nashik having following complaints but he didn't get relief anywhere so he was admitted in SMBT hospital for 12 days.

\section{$\mathrm{C} / \mathrm{O}$}

BL knee joint pain: 3 yrs.

BL knee joint swelling: 3 yrs.

Restricted movement of BL knee joint were having swelling \& restricted movements. X- ray of BL knee joint showed osteoarthritic changes and osteophytes.

He was diagnosed as a case of Januandhigatavata on the basis of clinical sign, symptom, X-ray, finding \& examination. Treatment was started after detailed history taking $\&$ examination of the patient.

\section{Examination of patient-}

Table A -

Ashtavidhanparikshana

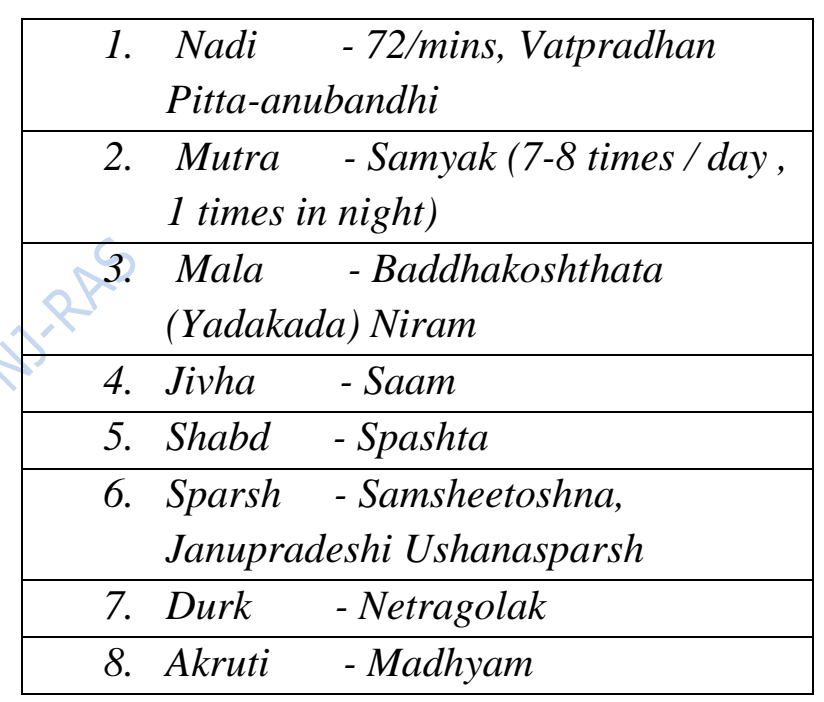

Table 1

Table B - Aturbalapramana Pariksha

\begin{tabular}{|c|c|}
\hline Pittanubandhi & $\begin{array}{l}\text { - Vatpradhan } \\
\text { hi }\end{array}$ \\
\hline 2. Vikruti & - Sandighatavata \\
\hline 3. Samhanan & - Madhyam \\
\hline 4. Sara & - Madhyam \\
\hline 5. Praman & - Madhyam \\
\hline 6. Satmyatah & - Avar \\
\hline 7. Satva & - Madhyam \\
\hline 8. Aaharshakt & - Madhyam \\
\hline
\end{tabular}

Table 2 


\section{Assessment Criteria -}

The improvement in the patient like reduction in pain, swelling, restricted movement score graded based on following scales.

\section{Pain (Shoola) -}

\section{$10 \mathrm{~cm}$ Visual Analogue Scale (Vas)}

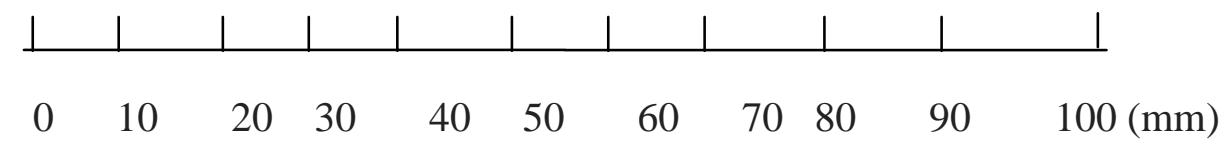

No pain

maximum pain

\section{Pidanasahatva (Tenderness) -}

\begin{tabular}{|c|c|c|}
\hline Sr. no. & $\begin{array}{c}\text { Severity of } \\
\text { Symptoms }\end{array}$ & Grade \\
\hline 1 & No tenderness & 0 \\
\hline 2 & $\begin{array}{c}\text { Patient says } \\
\text { tenderness }\end{array}$ & 1 \\
\hline 3 & Wincing of face & 2 \\
\hline 4 & $\begin{array}{r}\text { Wincing of face \& } \\
\text { withdrawal of } \\
\text { affecting part }\end{array}$ & 3 \\
\hline
\end{tabular}

Table - 3

\section{Shotha (Swelling)}

\begin{tabular}{|c|c|c|}
\hline Sr. no. & $\begin{array}{c}\text { Severity of } \\
\text { Symptoms }\end{array}$ & Grade \\
\hline 1 & No shotha & 0 \\
\hline 2 & Slight shotha & 1 \\
\hline 3 & Moderate shotha & 2 \\
\hline 4 & Severe shotha & 3 \\
\hline
\end{tabular}

Table - 4

\section{Crepitus}

\begin{tabular}{|c|l|c|}
\hline Sr. no. & $\begin{array}{l}\text { Severity of } \\
\text { Symptoms }\end{array}$ & Grade \\
\hline 1 & No Crepitus & 0 \\
\hline 2 & $\begin{array}{c}\text { omplained by patient } \\
\text { but not felt in }\end{array}$ & 1 \\
\hline
\end{tabular}

\begin{tabular}{|c|c|c|}
\hline & examination & \\
\hline 3 & Felt on examination & 2 \\
\hline 4 & $\begin{array}{c}\text { Felt \& heard on } \\
\text { examination }\end{array}$ & 3 \\
\hline
\end{tabular}

Table - 5

\section{Treatment Protocol -}

Patient is given both shaman \&

Panchakarma Chikitsa. Patient was given

following medicines followed by

Panchakarma \& got significant result.

Ayurvedic Management -

\begin{tabular}{|c|c|l|l|}
\hline $\begin{array}{c}\text { Sr. } \\
\text { No } \\
\cdot\end{array}$ & Medicine & Dose & Frequency \\
\hline 1 & Yograj Guggula & $2 \mathrm{Tab}$ & $\mathrm{BD}$ \\
\hline 2 & Mahavatvidhvansa & $2 \mathrm{Tab}$ & $\mathrm{BD}$ \\
\hline 3 & Dashmool Kwath & $40 \mathrm{ml}$ & $\mathrm{BD}$ \\
\hline 4 & avanbhaskar Churna & $3 \mathrm{gm}$ & $\mathrm{BD}$ \\
\hline 5 & $\begin{array}{c}\text { Swadishtha Virechan } \\
\text { churna }\end{array}$ & $3 \mathrm{gm}$ & $\mathrm{HS}$ \\
\hline
\end{tabular}

Table -6 


\section{Panchkarma Rx -}

- Sarvang Snehan \& Swedan is started after 3 days of oral medication.

- Janubasti was given after Snehan \& Swedan with Mahanarayn tail \& Sahachar Tail mixed oil.

- With above measures, Yogbasti Karma was given to patient for 8 days from $4^{\text {th }}$ day.

\begin{tabular}{|c|c|c|}
\hline 1 & $\begin{array}{l}\text { Day } 1 \text { - oral } \\
\text { medication }\end{array}$ & \\
\hline 2 & $\begin{array}{l}\text { Day } 2 \text { - oral } \\
\text { medication }\end{array}$ & \\
\hline 3 & $\begin{array}{l}\text { Day } 3 \text { - oral } \\
\text { medication }\end{array}$ & \\
\hline 4 & $\begin{array}{l}\text { Day } 4- \\
\text { Anuvasan } \\
\text { Basti }\end{array}$ & $\begin{array}{c}\text { Mahanarayan } \\
\text { Tail }+ \\
\text { Sahachar } \\
\text { Tail } \\
40 \mathrm{ml} \\
40 \mathrm{ml}\end{array}$ \\
\hline 5 & $\begin{array}{l}\text { Day } 5- \\
\text { Anuvasan } \\
\text { Basti }\end{array}$ & Same as above \\
\hline 6 & $\begin{array}{l}\text { Day 6- } \\
\text { Niruha }\end{array}$ & $\begin{array}{c}\text { Dashamoola } \\
\text { Niruha } \\
(960 \mathrm{ml})\end{array}$ \\
\hline 7 & $\begin{array}{l}\text { Day } 7- \\
\text { Anuvasan }\end{array}$ & $\begin{array}{r}\text { Mahanarayan } \\
\text { Tail }(80 \mathrm{ml})\end{array}$ \\
\hline 8 & $\begin{array}{l}\text { Day } 8- \\
\text { Niruha }\end{array}$ & $\begin{array}{c}\text { Dashamoola } \\
\text { Niruha }(960 \\
\text { ml })\end{array}$ \\
\hline 9 & $\begin{array}{l}\text { Day } 9- \\
\text { Anuvasan }\end{array}$ & $\begin{array}{r}\text { Mahanarayan } \\
\text { Tail }(80 \mathrm{ml})\end{array}$ \\
\hline 10 & Day $10-$ & Dashamoola \\
\hline
\end{tabular}

\begin{tabular}{|r|c|c|}
\hline & Niruha & Niruha $(960$ \\
& & ml) \\
\hline \multirow{2}{*}{11} & Day 11- & Mahanarayan \\
& Anuvasan & Tail \\
\hline
\end{tabular}

Preparation of Dashamoola Niruha Basti-

For prepration of Dashamoola Niruha basti 200 gms of Dashmool baharad was boiled $320 \mathrm{ml}$ of .ashmool Kwath.

Total Quantity of Niruha Basti - 960 ml

Kwath- $800 \mathrm{ml}$

Madhu- $25 \mathrm{ml}$

Dashamoola churna- 30 gms

Mahanarayan Tail-100ml

Saindhav- $5 \mathrm{gm}$

In the vessel, honey \& Saindhav are added, mixed well. After that warm oil is added, \& stirred well, then paste of drugs is added. After stirring well, Dashmool Kwath is added and then combined well $\&$ put in the bastiyantra.

\section{Prepation of Anuvasan:}

$$
\begin{array}{ll}
\text { Mahanarayan Tail - } & 120 \mathrm{ml} \\
\text { Shatpushpa Churna - } & 1 \mathrm{gm} \\
\text { Saindhav - } & 1 \mathrm{gm}
\end{array}
$$

Tail is heated indirectly. Shatapushpa Churna \& Dashmool Churna are added as prakshep. 


\section{Result}

As per objective assessment criteria, patient's sign \& were analyzed before treatment \& after treatment and during followed period. The result shown in the table as under

\begin{tabular}{|c|c|c|c|c|c|c|c|c|c|c|c|}
\hline $\begin{array}{r}\text { Sr. } \\
\text { n }\end{array}$ & Knee & \multicolumn{2}{|c|}{ Pain } & \multicolumn{2}{|c|}{ Tenderness } & \multicolumn{2}{|c|}{ Swelling } & \multicolumn{2}{|c|}{ Crepitus } & \multicolumn{2}{|c|}{ Total score } \\
\hline & & BT & $\mathrm{AT}$ & $\mathrm{BT}$ & AT & $\mathrm{BT}$ & $\mathrm{AT}$ & BT & $\mathrm{AT}$ & BT & $\mathrm{AT}$ \\
\hline 1 & $\begin{array}{l}\text { Rt. } \\
\text { K } \\
\text { ne } \\
\text { e }\end{array}$ & 40 & 20 & 2 & 1 & 2 & 1 & 2 & 2 & 46 & 24 \\
\hline 2 & $\begin{array}{l}\text { Lt. } \\
\text { kn } \\
\text { ee }\end{array}$ & 40 & 20 & 2 & 1 & 2 & 1 & 2 & 1 & 46 & 23 \\
\hline \multicolumn{10}{|c|}{ Total } & 92 & 47 \\
\hline
\end{tabular}

As per above table score was 92 at the time of admission \& if reduced to 47 at the time of discharge . It showed $52 \%$ relief in patient

\section{Discussion}

Sandhigatavata is a Nirupstambhit Vat vyadhi caused by Dhatukshay. With the age, Rukshata of Vat dosha increases and so increase the Dhatushaya. So Snehan is a given to the patient that reduced pain, braught back the Mardavata of joints. Swedan helps to reduce stiffness and provide flexibility of Sandhi. Janubasti reduces the Shool, Shabd and Shotha. It gives strength to the joint. Oral Ayurvedic medicines are Vathara, Shothhara, Shoolghna and Rasayan. In Janusandhigatavata, Vat Dosha is vitiated by Ruksha, laghu guna that is mitigated by Basti.

\section{REFERENCES:}

1. Charaksamhita, revised by Dhrudhabala with Ayurvedic Dipika commentary by Chakrapanidatta edited by Chaukhambha publications, Varanasi,2007.

2. Sushrutsamhita with the nibandhasangraha commentary of Dalhan edited by Yadavji Trikamji, published by Chaukhamabha publications, Varanasi, 2008.

3. Ashtang hridaya, with Sarvang sundar commentary of Hemadri, edited by Pt. Shastri, Chaukhambha publications, Varanasi.

4. Sharandhar Samhita, Purva khanda, 0/1-12, edited by Bramhanand Tripathi, Chaukhambha publications, Varanasi.

5. Vaidya H S Kasture, Ayurved Panchakarma Vigyan, Shri 
Vaidyanath Ayurved Bhavan LTD, Alhabad, reprint-2006.

6. Bhaishajya Ratnavali, Edited by Rajeshwar Dutta Shastri, Chaukhambha Sanskrit Santhan, Varanasi.
7. Harrisons Principle of Internal Medicine, $17^{\text {th }}$ editions $v o l / 1 \& 2$, 2008.

8. Davidsons principle \& practice of medicine, $20^{\text {th }}$ edition reprint, 2008.

9. Harsh Mohan Text Book of Pathology, 6th Edition, 2010.

Conflict of Interest: Non

Source of funding: Nil

Cite this article:

A Case Study of Janusandhigatavata (Osteoarthritis of knee joint)

Anushri Gokul Endait, Mayuri Santosh Nagre, Smita Madhav Kanawade

Ayurlog: National Journal of Research in Ayurved Science- 2020; (8) (5):01- 06 